\title{
Fatores associados à adesão ao uso de órteses de membro superior sob diferentes perspectivas
}

\author{
Factors associated with accession to the use of upper member orthosis under different \\ perspectives
}

\author{
Factores asociados a la adhesión al uso de ortesis de miembros superiores bajo \\ diferentes perspectivas
}

Simone Stein Siqueira ${ }^{1 *}$, Heloísa Helena Motta Bandini ${ }^{1}$.

\begin{abstract}
RESUMO
Objetivo: Identificar fatores associados na adesão dos usuários ao uso de órtese de membro superior no serviço de um Centro Especializado em Reabilitação (CER) na visão do profissional, do paciente e dos discentes dos cursos de fisioterapia e terapia ocupacional. Métodos: Pesquisa descritiva com abordagem qualiquantitativa. Participaram: 11 profissionais, 30 discentes, 9 usuários. Foram aplicados formulários estruturados. Resultados: Os resultados foram avaliados nas categorias: orientação, adesão e desafios. Falta de compreensão do objetivo da órtese, pontos de pressão, dor, desconforto foram evidenciados como preponderantes no quesito adesão, na percepção dos profissionais e discentes; enquanto limitação no desempenho das atividades de vida diária, na percepção dos usuários. Desafios: custo e dificuldade em adquirir os materiais, pelo setor público. Na percepção dos discentes, aponta-se a necessidade de promover melhora na integração ensino, pesquisa e assistência em saúde. Conclusão: Verificou-se que os fatores preeminentes associados à adesão ao uso de órtese de membro superior foram diversificados na percepção dos sujeitos da pesquisa. Entretanto, os desafios foram similares entre os sujeitos.
\end{abstract}

Palavras-chave: Tecnologia assistiva, Órtese, Mão.

\section{ABSTRACT}

Objective: To identify factors associated with users' accession to the use of upper member orthosis in the service of a Specialized Rehabilitation Center (CER) in the view of the professional, the patient and the students of the physiotherapy and occupational therapy courses. Methods: Descriptive research with a qualitative and quantitative approach. Participated: 11 professionals, 30 students, 9 users. Structured forms were applied. Results: The results were evaluated in the categories: orientation, adherence and challenges. Lack of understanding of the purpose of the orthosis, pressure points, pain, discomfort were evidenced as preponderant in terms of adherence, in the perception of professionals and students; while limiting the performance of activities of daily living, in the perception of users. Challenges: cost and difficulty in acquiring the materials, by the public sector. In the perception of the students, the need to promote improvement in the integration of teaching, research and health care is pointed out. Conclusion: It was found that the prominent factors associated with adherence to the use of upper limb orthosis were diverse in the perception of the research subjects. However, the challenges were similar between the subjects.

Keywords: Assistive technology, Orthosis, Hand.

\section{RESUMEN}

Objetivo: Identificar factores asociados a la adherencia de los usuarios al uso de órtesis de miembro superior en el servicio de un Centro de Rehabilitación Especializado (CER) en la visión del profesional, el paciente y los estudiantes de los cursos de fisioterapia y terapia ocupacional. Métodos: Investigación descriptiva con enfoque cualitativo y cuantitativo. Participaron: 11 profesionales, 30 estudiantes, 9 usuarios. Se aplicaron formas estructuradas. Resultados: Los resultados se evaluaron en las categorías: orientación, adherencia y desafíos. La

${ }^{1}$ Universidade Estadual de Ciências da Saúde de Alagoas (UNCISAL), Maceió - AL.

*E-mail: simone.stein@uncisal.edu.br 
falta de comprensión del propósito de la órtesis, puntos de presión, dolor, malestar se evidenció como preponderantes en cuanto a adherencia, en la percepción de profesionales y estudiantes; al tiempo que limita el desempeño de las actividades de la vida diaria, en la percepción de los usuarios. Desafíos: costo y dificultad de adquisición de materiales por parte del sector público. En la percepción de los estudiantes se señala la necesidad de promover la mejora en la integración de la docencia, la investigación y la atención de la salud. Conclusión: Se encontró que los factores destacados asociados a la adherencia al uso de ortesis de miembro superior fueron diversos en la percepción de los sujetos de investigación. Los desafíos fueron similares entre los sujetos.

Palabras clave: Tecnología de asistencia, Ortesis, Mano.

\section{INTRODUÇÃO}

A partir da implantação do Sistema Único de Saúde (SUS) em 1988 o acesso à assistência de saúde a todo cidadão passou a ocorrer nos três níveis: prevenção, tratamento e reabilitação (BRASIL, 1988). Desde então, leis têm sido estabelecidas para garantir à acessibilidade aos serviços, bem como a disponibilidade de recursos de Tecnologia Assistiva de modo a proporcionar condições às pessoas com deficiência para que consigam autonomia e independência em todos os âmbitos de sua vida (BRASIL, 2004; BRASIL, 2009).

A tecnologia assistiva visa promover a inclusão e integração das pessoas com deficiência e possibilita potencializar as habilidades funcionais, ou seja, permite melhoria da qualidade de vida do usuário (BRASIL, 2009). Neste contexto as órteses são consideradas como tecnologia assistiva, pois objetivam a melhora funcional do paciente. Desta forma, a órtese de membro superior tem seu papel definido na reabilitação e pode ser utilizada como recurso terapêutico.

No Brasil, os serviços de reabilitação além de oferecerem atendimento especializado às pessoas com deficiência, utilizam de dispositivos da tecnologia assistiva, como as órteses, a fim de favorecer a funcionalidade e autonomia aos indivíduos, sendo aplicadas à um segmento corporal para assistir (restaurar ou substituir) em uma função comprometida, proporcionando mudanças anatômicas e funcionais; consequentemente alterando a participação em papéis desempenhados e considerados importantes para cada indivíduo, como: social, familiar, lazer, labor ou outro (FESS EE, 2002; AGNELLI LB e TOYODA CY, 2003; SOUZA MA, et al., 2015; SCHOFIELD KA e SCHWARTZ DA, 2020).

Órtese é definida como um dispositivo pré-fabricado ou feito sob medida, aplicando forças às estruturas biológicas de um segmento do corpo comprometido por lesão aguda, trauma cumulativo, doença, intervenção cirúrgica, anomalia congênita ou mudanças degenerativas (MCKEE P e RIVARD A, 2005). É primordial enfatizar que a confecção da órtese deve ser fundamentada na avaliação de desempenho funcional e o contexto de vida do paciente, no respeito aos princípios biomecânicos e funcionais, no caso específico de membro superior, como: posição funcional da mão, anatomia (preservação aos arcos e pregas palmares), distribuição de pressão, equilíbrio de forças, rigidez e resistência do material, resposta dos tecidos ao estresse, na escolha do material para remodelagem, flexibilidade, condições de uso e aceitação do paciente (FERRIGNO IS, 2009; LEDE PV, 2002).

Os benefícios do tratamento com órteses são diretamente proporcionais à adesão do paciente ao uso (JOSEPH M, et al., 2018). Em lesões agudas de membros superiores, a não aderência ao uso da órtese pode comprometer a eficácia do tratamento, propiciar viés na eficácia da intervenção e aumentar o risco de incapacidade (O'BRIEN L, 2010). Entre os fatores que interferem na adesão ao uso de órtese encontramse: condição socioeconômica, crença cultural, redução de motivação, aparência e conforto da órtese (JOSEPH M, et al., 2018).

Salienta-se que a órtese desempenha papel integrador no programa de reabilitação, mas não substitui outras intervenções. Por se tratar de uma parte do processo de reabilitação o uso da órtese requer acompanhamento periódico para que haja constante reavaliação de seu uso, e quando necessária modificação do modelo e/ou uso ou até mesmo a suspensão. O terapeuta deve promover a articulação entre o objetivo e a aceitação da órtese pelo cliente a fim de obter o uso como prescrito, bem como os ganhos pretendidos (SCHOFIELD KA e SCHWARTZ DA, 2020). 
A presente pesquisa teve como objetivo identificar fatores associados à adesão dos usuários ao uso de órtese de membro superior no serviço de um Centro Especializado em Reabilitação na cidade de Maceió/Alagoas, verificando quais as dificuldades e desafios são mais frequentes tanto na visão do profissional, como do paciente/ cuidador e dos discentes (estagiários).

\section{MÉTODOS}

Pesquisa exploratória, descritiva de abordagem qualiquantitativa desenvolvida no Centro Especializado de Reabilitação (CER) na cidade de Maceió - Alagoas, tendo um total de 50 participantes. Os critérios de inclusão foram utilizados de acordo com as categorias dos sujeitos do estudo. Na categoria profissional, foram incluídos todos os profissionais, docentes e técnicos, que atuavam no serviço CER, local da pesquisa, e trabalhavam com confecção e/ou prescrição de órteses para membro superior.

A categoria usuário contemplou cuidadores ou pacientes que faziam uso de órtese de membro superior e estavam em atendimento no serviço, na fisioterapia e/ou terapia ocupacional. Na categoria discentes, teve como critérios ser aluno do último ano dos cursos de fisioterapia e terapia ocupacional em estágio supervisionado obrigatório na área de reabilitação física, no local da pesquisa. $O$ trabalho foi submetido à Plataforma Brasil, analisado pelo Comitê de Ética em Pesquisa- CEP da Universidade Estadual de Ciências da Saúde de Alagoas (UNCISAL), de acordo com o certificado de apresentação para apreciação ética CAEE número 85104118.0.0000.5011, obtendo aprovação mediante o parecer número 2.617.955, em conformidade com a resolução № 466/2012.

O estudo foi disposto em quatro fases, sendo a primeira, a elaboração do material de pesquisa: questionários para os profissionais, os discentes e formulário para paciente/cuidador com linguagem adequada a cada grupo de sujeitos. $O$ instrumento de coleta possuía questões objetivas e abertas. Foi realizado teste piloto. Na segunda fase, ocorreu a coleta de dados. Os sujeitos puderam escolher a forma conveniente para responderem os instrumentos de pesquisa: escrita ou através da gravação de áudio. A terceira fase constituiu a análise dos dados e discussão de acordo com as categorizações por tema dos questionários. Para a análise dos dados subjetivos, utilizou-se a técnica da análise de conteúdo conforme Bardin L (2011).

Esse processo permitiu a formulação de três categorias temáticas, elaboradas a priori, tomando como base o instrumento da pesquisa: orientações (dadas ou recebidas quanto à indicação, uso, manuseio e preservação da órtese); adesão (interferência no uso da órtese); desafios na prescrição e confecção. Foram estabelecidas as três categorias para o grupo de profissionais e discentes e apenas duas categorias (orientação e adesão) para os usuários. A quarta fase proporcionou a elaboração do material educativo.

\section{RESULTADOS E DISCUSSÃO}

Os sujeitos da pesquisa encontram-se distribuídos em três grupos: usuários $(U)$, profissionais $(P)$ e discentes (D) (Tabela 1, Tabela 2, Tabela 3).

Tabela 1 - Caracterização dos Profissionais Participantes da Pesquisa, $n=11$.

\begin{tabular}{c|c}
\hline VARIÁVEL & CARACTERIZAÇÃO DA POPULAÇÃO \\
\hline Média de tempo de graduação & 16,9 anos \\
Sexo Feminino & $100 \%$ \\
Sexo Masculino & $0 \%$ \\
Pós Graduação (Mestrado) & $45 \%$ \\
Pós Graduação (Especialização) & $55 \%$ \\
Graduação (Terapia Ocupacional) & $82 \%$ \\
Graduação (Fisioterapia) & $18 \%$ \\
Orientação (oral) & $64 \%$ \\
Orientação (oral e escrita) & $36 \%$ \\
Ação profissional (docente) & $64 \%$ \\
Ação profissional (técnico) & $36 \%$ \\
Órtese (indicação) & $64 \%$ \\
Órtese (indicação e confecção) & $36 \%$ \\
\hline
\end{tabular}

Fonte: Siqueira SS e Bandini HHM, 2020. 
Tabela 2 - Caracterização dos Discentes Participantes da Pesquisa, $n=30$.

\begin{tabular}{c|c}
\hline VARIÁVEL & CARACTERIZAÇÃO DA POPULAÇÃO \\
\hline Sexo Feminino & $93 \%$ \\
Sexo Masculino & $7 \%$ \\
Graduação (Fisioterapia) & $30 \%$ \\
Graduação (Terapia Ocupacional) & $70 \%$ \\
\hline
\end{tabular}

Fonte: Siqueira SS e Bandini HHM, 2020.

No grupo dos discentes, todos cursavam o último ano dos cursos de fisioterapia e terapia ocupacional, em estágio supervisionado obrigatório na área de reabilitação física.

Tabela 3 - Caracterização dos Usuários de Órtese Participantes da Pesquisa, n=9.

\begin{tabular}{c|c}
\hline VARIÁVEL & CARACTERIZAÇÃO DA POPULAÇÃO \\
\hline Média de idade (em anos) & 46,44 \\
Sexo Feminino & $67,0 \%$ \\
Sexo Masculino & $33,0 \%$ \\
Escolaridade (Fundamental I Incompleto) & $33,33 \%$ \\
Escolaridade (Fundamental I Completo) & $22,22 \%$ \\
Escolaridade (Médio) & $22,22 \%$ \\
Escolaridade (Superior) & $22,22 \%$ \\
Tratamento (Fisioterapia e Terapia Ocupacional) & $33,0 \%$ \\
Tratamento (Terapia Ocupacional) & $67,0 \%$ \\
\hline
\end{tabular}

Fonte: Siqueira SS e Bandini HHM, 2020.

Com relação aos usuários, o tempo médio de uso da órtese são 16,9 meses, o que permite aos usuários boa capacidade para refletir e perceber alterações com o uso do equipamento. A indicação da órtese foi realizada por terapeuta ocupacional $(89 \%)$ e médico $(11 \%)$, enquanto a confecção foi feita por terapeuta ocupacional $(89 \%)$ e fisioterapeuta $(11 \%)$. As órteses em uso foram confeccionadas artesanalmente no serviço em que estavam em atendimento.

Os dados coletados divergem da pesquisa realizada por Agnelli LB e Toyoda CY (2003) em que a indicação/prescrição da órtese, na maioria das instituições, era realizada por médicos e o terapeuta ocupacional realizava apenas a confecção e acompanhamento dos casos. Pode-se inferir que a organização do CER, com maior número de profissionais da área de reabilitação (fisioterapeutas e terapeutas ocupacionais) com autonomia nas ações competentes à cada categoria, bem como o maior acesso dos pacientes aos serviços de reabilitação prestados proporcionou maior atuação destes profissionais na prescrição de órteses do membro superior. Também, merece destaque a formação (graduação e continuada) destes profissionais para o desempenho destas atividades.

Em relação às orientações sobre o uso, a conservação e higiene da órtese, $87 \%$ dos discentes informaram realizar, sendo $77 \%$ na forma oral e $10 \%$ na forma oral e escrita. Entre os docentes $64 \%$ informaram que realizam somente na forma oral e $36 \%$ na forma oral e escrita. Tanto profissionais como discentes realizam esclarecimentos sobre ação, uso, material, cuidado e higiene da órtese, como citado nas falas:

"Qual o objetivo da órtese para o caso específico do paciente, como ela irá atuar. Tempo de uso e quando não deve usar, possíveis dificuldades que possam surgir, como pontos de pressão devendo suspender o uso e informar imediatamente ao profissional para que ajustes sejam feitos. Também oriento que a dor é um sinal de alerta, por isso devem informar ao profissional caso ocorra. Também informo sobre a conservação da órtese, no caso de termoplástico, quanto à temperatura e limpeza." (P11) 


\begin{abstract}
"Os esclarecimentos são feitos de forma oral. Explicando ao paciente tipo de órtese, a forma que deve ser usada e o que o mau uso da órtese pode acarretar na disfunção presente." (D2)
\end{abstract}

Constata-se que as orientações dadas aos usuários pelos profissionais e discentes são predominantemente na forma oral, contribuindo para a menor assimilação, devido às dificuldades sócio econômicas apresentadas pela população atendida. Verificou-se a falta de um material de apoio para consulta posterior e reforço das informações.

Ao questionar os pacientes sobre as orientações recebidas quanto ao manuseio, preservação e higiene da órtese, percebe-se que há dúvidas e pouca informação absorvida por eles. Verifica-se que $78 \%$ dos usuários relatam que recebeu orientação quanto ao tempo de uso diário da órtese, mas não sabem especificar qual o tempo específico para uso. Dos $89 \%$ que informam ter recebido orientação sobre higiene, $25 \%$ descrevem o uso de produtos abrasivos para limpeza da órtese, o que é contraindicado para o material termoplástico de baixa temperatura.

Quanto à colocação e retirada das órteses, os usuários relatam realizar este procedimento de forma fácil (55\%) ou razoável dificuldade (45\%), não necessitando de auxílio de terceiros, o que favorece a adesão. Fatores como facilidade de higiene, conforto, durabilidade, simplicidade do uso e compreensão da ação interferem no uso da órtese (HOHMANN P e CASSAPIAN MR, 2011). Ainda na orientação quanto à indicação, os usuários relatam conhecer a ação da órtese no seu agravo e $89 \%$ classificam a gravidade como sendo de média à severa. O usuário (U4) reconhece que a órtese proporciona posição funcional do membro superior, favorecendo a função.

“...Sofri a lesão do nervo radial, perdendo o movimento de punho e extensão de dedos (...) ela faz a melhora na extensão dos dedos e na sustentação do punho."

Enquanto recurso de tecnologia assistiva, a órtese foi reconhecida por cada usuário, de acordo com as especificidades, como: promovendo posicionamento do membro superior, prevenindo deformidades, fornecendo suporte e alinhamento das articulações, viabilizando a estabilidade muscular, resgatando a função manual que estava comprometida ou perdida conforme sugere a literatura, alterando inclusive 0 desempenho ocupacional (AGNELLI LB e TOYODA CY, 2003; MCKEE P e RIVARD A, 2005; SILVA TSS e MASSA LDB, 2015).

De acordo com McKee P e Rivard A (2005), uma órtese promove alteração nos tecidos moles, ossos, articulações, como também no desempenho ocupacional. Assim, a interação com o paciente durante todo o processo, fornecendo orientações claras e objetivas possibilitam melhor resultado.

De acordo com a World Health Organization (2003), a adesão é influenciada por cinco dimensões: socioeconômica, relacionada ao paciente, ao tratamento, sistema de saúde e à própria condição de saúde. Cada um desses fatores deve ser considerado quando se almeja promover adesão à uma determinada intervenção. Não há estratégias de intervenção específica eficaz para todos os pacientes. No entanto, é de comum acordo que os pacientes devem ser participativos, pois a melhoria da adesão a um tratamento é um processo ativo e frequente. $O$ resultado do tratamento de lesões musculoesqueléticas agudas depende da adesão do paciente ao uso da órtese (JOSEPH M, et al., 2018; O'BRIEN L, 2010).

Os fatores que interferem na adesão ao uso da órtese, na perspectiva profissional, destacam-se: a falta de compreensão do objetivo da órtese pelo paciente, desconforto, aceitação social. Na percepção discente, as dificuldades mais frequentes apresentadas pelos pacientes são, em ordem decrescente: desconforto, dor, falta de compreensão do objetivo da órtese e pontos de pressão, dificuldade de colocação e retirada, coceira e em último lugar, estética, afecção na pele e ajuste biomecânico. Discentes e profissionais diferem entre si na percepção da hierarquia das dificuldades indicadas na adesão, sendo o desconforto o fator mais citado pelos discentes e não citado pelos profissionais.

O'Brien L (2010), na revisão sistemática realizada, encontrou apenas um estudo em que desconforto foi considerado como fator relevante para o não uso da órtese, fato que corrobora com a percepção dos profissionais desta pesquisa. 
Paterson AMJ, et al. (2014) destacam como complicações na aderência do paciente ao uso da órtese: o desconforto causado por pontos de pressão, estética pobre, peso, limitação da função e comprometimento do desempenho na realização de atividades de vida diária, entre outros. Os resultados, nesta pesquisa, demonstram estes mesmos fatores causando interferência na adesão na percepção dos profissionais e discentes, exceto peso da órtese. O desconforto é fator preponderante para os discentes, entretanto usuários e profissionais não priorizaram. MacKee P e Rivard A (2004) enfatizam que bons resultados no uso de órtese ocorrem quando há conforto, leveza, estética e compreensão sobre o uso.

Conhecer as necessidades, preferências e predisposições individuais, incluir estilo de vida, comportamentos, expectativas e habilidades, realizar avaliação permanente, compartilhar os resultados com o paciente são preponderantes para o êxito no uso de tecnologia assistiva (ALVES ACJ, 2017). Profissionais e discentes atestam em suas falas a realização destas ações.

"Melhorar as informações sobre a importância, ação da órtese, reforçando constantemente. Fazer acompanhamento sistemático, mensurar resultados e apresentá-los de forma compreensível para o paciente. Ser um "bom ouvinte", ou seja, escutar o paciente em suas dúvidas e até mesmo em possíveis soluções em relação à órtese." (P11)

"A orientação adequada sobre os objetivos da utilização da órtese, as adequações da mesma e os benefícios advindos pela utilização frequente dela se explicados de maneira simples e na linguagem que o paciente compreenda possivelmente contribuirá na motivação do paciente para adesão à órtese." (D15)

Há concordância que o conhecimento do paciente sobre a ação da órtese, bem como sua manutenção e conservação interferem na utilização diária apropriada, na conservação, durabilidade e manutenção, promovendo maior adesão ao uso, para isto é necessário esclarecimento adequado à sua realidade e compreensão.

"Fazendo a orientação sobre a necessidade e os benefícios da utilização da órtese, assim como realizar o treinamento de forma simples e de fácil compreensão para a utilização do mesmo; facilitar o acesso ao serviço de órtese." (P04)

Os sujeitos da pesquisa reconheceram que no processo de reabilitação com uso de órtese é imprescindível conhecer as necessidades (físicas e psicossociais), preferências do paciente, ter uma comunicação adequada de escuta e transmissão de informações (a atitude do terapeuta desde o acolhimento e até o encorajamento para seguir as recomendações), desenvolver a relação terapeutapaciente, tornar o indivíduo coparticipativo inclusive na realização do programa domiciliar.

Faz-se necessário entender os componentes primários que exercem influência na satisfação e uso da tecnologia assistiva, a saber: o meio ambiente e social no qual o usuário irá interagir (seu contexto); as necessidades (incluindo tipo de lesão e/ou condição clínica), preferências, motivação e expectativas do usuário e as atividades exercidas pelo mesmo; as funções e características da tecnologia que atendam a demanda do usuário em questão, pois a adesão é fundamental no processo de reabilitação (MACKEE $P$ e RIVARD A, 2004; COOPER RG e EDGETT SJ, 2008; MATOZZO TR, 2016; GRADIM LCC e PAIVA G, 2018).

O uso da órtese foi considerado satisfatório no julgamento dos usuários por promover melhora na posição funcional, evitar deformidade nas articulações, propiciar equilíbrio muscular, reduzir quadro álgico, alterar o desempenho funcional, reforçar a segurança. Estes fatores culminaram na melhora da qualidade de vida propiciando maior bem-estar e elevação da autoestima de acordo com o relato dos entrevistados.

Entre os achados de Joseph $\mathrm{M}$, et al. (2018) quanto à satisfação dos usuários de órtese de membro superior, destacam-se três qualidades como prioridades: conforto (81\%), eficácia $(75 \%)$ e facilidade de uso (74\%). Ressalta-se que os achados da pesquisa, aqui apresentada, obtiveram a eficácia em primeiro lugar, em seguida conforto e por último a facilidade de uso. 
Em relação à interferência no uso, somente $55,5 \%$ dos usuários relatam ter dificuldades, sendo estas: alteração na realização das atividades de vida diária por limitação: restrição dos movimentos (75\%) e pontos de pressão (25\%). Cabe destacar que os usuários que relatam estas dificuldades fazem uso de órtese para corrigir e/ou evitar deformidades ao nível das articulações da mão e dedos, devido ao agravo ser crônico.

Sendo o comprometimento na realização de atividades de vida diária fator relevante para alguns, devese considerar o desempenho nas atividades de vida diária ao escolher o modelo de órtese a fim de que não haja restrição da funcionalidade (O'BRIEN L, 2010; COSTA CR, et al., 2015; NEMATI Z, et al., 2016).

Santos RF, et al. (2017) relata que apesar das tecnologias assistivas proporcionarem funcionalidade há uma interface com o estigma. Fato este que pode contribuir para a não aceitação e uso. Cabe destacar que os usuários entrevistados relatam que a funcionalidade superou qualquer preconceito existente, percebendo inclusive a órtese como parte de seu visual, conforme o relato do usuário abaixo:

"(...) A gente estranha, é um impacto no primeiro momento. Aí depois você olha pra ela e começa a ter carinho. E eu tava refletindo agora, que pra mim o uso da órtese é usar um acessório como mulher, um acessório íntimo. Eu não posso sair sem aquela, o acessório íntimo. Então, a órtese, eu sinto dificuldade. Hoje, olhar para a minha mão e não perceber que estou com a órtese, é como se eu tivesse sem algo que é indispensável. Entende? Ela hoje tornou-se minha amiga íntima. Então, não estar com ela, falta alguma coisa." (U6)

Em relação aos desafios encontrados na prescrição de órteses, $64 \%$ dos profissionais consideram a restrição e falta de material como sendo fator preponderante, $18 \%$ dificuldade em atender a necessidade específica do paciente, $9 \%$ em indicar profissional capacitado para confeccionar a órtese. As dificuldades e desafios encontrados pelos profissionais na confecção de órteses encontram-se pautados, novamente, na restrição e falta de material $(46 \%)$ e $36 \%$ não realiza a confecção no serviço. Outro fator destacado é o tempo disponível para esta ação, pois por ser tratar de local público e demanda alta, há interferência na ação profissional.

Os achados desta pesquisa indicam dificuldades semelhantes aos achados de Agnelli LB e Toyoda CY (2003) em relação aos desafios na prescrição e confecção de órteses, sendo ressaltada pelos autores a burocracia do setor público na aquisição do material. Os desafios e dificuldades encontrados na prescrição e confecção de órteses pelos discentes são similares aos dos profissionais, sendo ainda sugerido o baixo conhecimento nos anos anteriores da graduação como sendo um fator limitante a estas ações.

Evidencia-se que na formação acadêmica, nos anos anteriores ao estágio, há necessidade da melhora da integração entre teoria e prática a fim de proporcionar no decorrer da vida universitária a formação de profissionais reflexivos, críticos, ativos no processo de aprendizagem, que busquem soluções e estratégias para intervir na realidade propiciando autonomia para o futuro profissional (MERIGHI MAB, et al., 2014; SCHOFIELD KA e SCHWARTZ DA, 2020).

Os dados da pesquisa corroboram com os achados de McKee P e Rivard A (2005) que considera que a abordagem centrada no paciente lhe possibilita compreender a indicação da órtese, bem como uso, cuidado e necessidade de acompanhamento do terapeuta. Cabe destacar que a interação terapeuta-cliente durante todo o processo (avaliação, confecção e intervenção) possibilita a otimização dos resultados.

É imprescindível que o profissional compreenda a satisfação e as expectativas do cliente em relação à órtese para que possa adequá-la ao propósito terapêutico. O terapeuta deve promover a articulação entre o objetivo e a aceitação da órtese pelo cliente a fim de obter o uso como orientado, bem como os ganhos pretendidos (SCHOFIELD KA e SCHWARTZ DA, 2020).

Apesar dos serviços de reabilitação possuírem um aparato legislativo para garantir o acesso à tecnologia assistiva, o custo e a dificuldade em adquirir os materiais, pelo setor público, foram apontados como os desafios preponderantes para prescrição e confecção das órteses. 


\section{CONCLUSÃO}

Os resultados deste estudo apontaram que os fatores preeminentes associados à adesão ao uso de órtese de membro superior foram diversificados na percepção dos sujeitos da pesquisa, sendo a funcionalidade no desempenho de atividades de vida diária fator de destaque pelos usuários e a escassez de conhecimento do usuário em relação à órtese e sua função como decisivos na adesão nas perspectivas do profissional e do discente. Entretanto, os desafios foram similares entre os sujeitos, sendo enfatizada a dificuldade na obtenção de materiais como fator dominante. Os dados desta pesquisa evidenciam a importância da orientação dada ao usuário no uso e cuidado com a órtese de membro superior e demonstra a necessidade do diálogo entre profissionais, discentes e pacientes promovendo reflexão e participação ativa no processo de reabilitação.

\section{REFERÊNCIAS}

1. AGNELLI LB, TOYODA CY. Estudo de materiais para a confecção de órtese e sua utilização prática por terapeutas ocupacionais no Brasil. Cadernos de Terapia Ocupacional da UFSCar, 2003; 11(2): 83-94.

2. ALVES ACJ. Avaliação de tecnologia assistiva predisposição ao uso: ATD PA Br: versão brasileira. Brasília: Editora Universidade de Brasília, 2017; 37p.

3. BARDIN L. Análise de conteúdo. São Paulo: Edições 70, 2011; 280p.

4. BRASIL. Constituição Federal. República Federativa do Brasil de 1988. Brasília, DF: Senado Federal, 1988.

5. BRASIL. Decreto № 5.296, de 2 de dezembro de 2004. Regulamenta as Leis nos 10.048, de 8 de novembro de 2000, que dá prioridade de atendimento às pessoas que especifica, e 10.098, de 19 de dezembro de 2000, que estabelece normas gerais e critérios básicos para a promoção da acessibilidade das pessoas portadoras de deficiência ou com mobilidade reduzida, e dá outras providências. Diário Oficial da União, Poder Executivo, Brasília, DF, 3 dez. 2004.

6. BRASIL. Subsecretaria Nacional de Promoção dos Direitos da Pessoa com Deficiência. Comitê de Ajudas Técnicas. Tecnologia Assistiva. Brasília: CORDE, 2009; 138 p.

7. COOPER RG, EDGETT SJ. Maximizing productivity in product innovation. Research-Technology Management, 2008; 51(2): 47-58.

8. COSTA CR, et al. Dispositivo de Tecnologia Assistiva: fatores relacionados ao abandono. Cadernos de Terapia Ocupacional UFSCar, 2015; 23(3): 611-624.

9. FESS EE. A History of Splinting: To Understand the Present, View the Past. Journal of Hand Therapy, 2002; 15(2): 97-132.

10. FERRIGNO ISV. Eletromiografia do membro superior no uso do computador: estudo comparativo entre duas órteses de punho. Dissertação (Doutorado em Cirurgia) - Faculdade de Ciências Médicas. Universidade Estadual de Campinas. Campinas, 2009; 99p.

11. GRADIM LCC, PAIVA G. Modelos de órteses para membros superiores: uma revisão da literatura. Cadernos Brasileiros de Terapia Ocupacional, 2018; 26(2): 479-488.

12. HOHMANN P, CASSAPIAN MR. Adaptações de baixo custo: uma revisão de literatura da utilização por terapeutas ocupacionais brasileiros. Revista de Terapia Ocupacional da Universidade de São Paulo, 2011; 22(1): 10-18.

13. JOSEPH M, et al. A survey of client experiences with orthotics using the QUEST 2.0. Journal of Hand Therapy, 2018; 31(4): 538-543.

14. LEDE PV. Minimalistic Splint Design. Journal of Hand Therapy, 2002; 15(2): 192-201.

15. MATTOZO TR. Tecnologia assistiva: identificação dos requisitos do produto de órteses para membros inferiores: uma visão a partir das percepções dos usuários. Dissertação (Mestrado em Design) - Centro de Comunicação e Expressão. Universidade Federal de Santa Catarina, Florianópolis, 2016; 138 p.

16. MCKEE P, RIVARD A. Orthoses as enablers of occupation: client-centered splinting for better outcomes. Canadian Journal Occupational Therapy, 2005; 71(5): 306-314.

17. MERIGHI MAB, et al. Ensinar e aprender no campo clínico: perspectiva de docentes, enfermeiras e estudantes de enfermagem. Revista Brasileira de Enfermagem, 2014; 67(4): 505-511.

18. NEMATI Z, et al. The effect of new dynamic splint in pinch strength in De Quervain syndrome: a comparative study. Disability and Rehabilitation: Assistive Technology, 2016; 12(5): 457-461.

19. O'BRIEN L. Adherence to therapeutic splint wear in adults with acute upper limb injuries: a systematic review. Journal of Hand Therapy, 2010; 15(1): 3-12.

20. PATERSON AMJ, et al. Computer-Aided Design to Support Fabrication of Wrist Splints Using 3D Printing: A feasibility study. Hand Therapy, 2014; 19(4): 102-113.

21. SANTOS RF, et al. Tecnologia assistiva e suas relações com a qualidade de vida de pessoas com deficiência. Revista de Terapia Ocupacional da Universidade São Paulo, 2017; 28(1): 54-62.

22. SCHOFIELD KA, SCHWARTZ DA. Teaching orthotic design and fabrication content in occupational therapy curricula: Faculty perspectives. Journal of Hand Therapy, 2020; 33(1):119-126.

23. SILVA TSS, MASSA LDB. A utilização de órteses de membro superior em pacientes com artrite reumatoide: uma revisão de literatura no campo da terapia ocupacional. Cadernos de Terapia Ocupacional da UFSCar, 2015; 23(3): 647-659.

24. SOUZA M, et al. Effect of using orthoses on prolonging ambulation in patients with Duchenne Muscular Dystrophy: review of literature. Acta Fisiátrica, 2015; 22(3): 155-159.

25. WORLD HEALTH ORGANIZATION. Adherence to long-term therapies: evidence for action. Geneva: World Health Organization, 2003. Disponível em: https://apps.who.int/iris/handle/10665/42682. Acesso em: 20 jan. 2019. 